Annals of Pure and Applied Mathematics

Vol. 15, No. 1, 2017, 25-40

ISSN: 2279-087X (P), 2279-0888(online)

Published on 11 December 2017

Annals of

www.researchmathsci.org

DOI: http://dx.doi.org/10.22457/apam.v15n1a3

Pure and Applied

Mathematics

\title{
Solution and Generalized Ulam-Hyers Stability of a $n$ - Dimensional Additive Functional Equation in Banach Space and Banach Algebra: Direct and Fixed Point Methods
}

\author{
M.Arunkumar ${ }^{1}$, P.Agilan ${ }^{2}$ and S.Ramamoorthy ${ }^{3}$ \\ ${ }^{1}$ Department of Mathematics, Government Arts College \\ Tiruvannamalai - 606 603, TamilNadu, India. \\ e-mail:annarun2002@yahoo.co.in \\ ${ }^{2}$ Department of Mathematics, Jeppiaar Institute of Technology \\ Sriperumbudur, Chennai - 631 604, Tamil Nadu, India. \\ e-mail:agilram@gmail.com \\ ${ }^{3}$ Department of Mathematics, Arunai Engineering College, \\ Tiruvannamalai - 606 603, TamilNadu, India. \\ e-mail:ramsdmaths@yahoo.com
}

Received 1 November 2017; accepted 8 December 2017

Abstract. In this paper, the authors investigate the general solution and generalized Ulam - Hyers stability of a new type of $\mathrm{n}$-dimensional additive functional equation

$$
\begin{aligned}
f\left(\sum_{k=1}^{n} k x_{k}\right)+\sum_{l=2}^{n} f\left(\sum_{k=1, k \neq l}^{n} k x_{k}-l x_{l}\right)+f\left(x_{1}-\sum_{k=2}^{n} k x_{k}\right) \\
=(n+1) f\left(x_{1}\right)+(n-3) \sum_{k=2}^{n} k f\left(x_{k}\right)
\end{aligned}
$$

with $n>3$ in Banach space and Banach Algebra using direct and fixed point methods.

Keywords: Additive functional equation, Generalized Ulam-Hyers stability, Banach Space, Banach Algebra, Fixed point.

AMS Mathematics Subject Classification (2010): 39B52, 39B82

\section{Introduction}

The stability problem of functional equations originated from a question of Ulam [41] concerning the stability of group homomorphisms. Hyers [25] gave a first affirmative partial answer to the question of Ulam for Banach spaces. Hyers' theorem was generalized by Aoki [2] for additive mappings and by Rassias [34] for linear mappings by considering an un-bounded Cauchy difference. The paper of Rassias [34] has provided a lot of influence in the development of what we call generalized Ulam stability of functional equations. In 1982, Rassias [17] followed the innovative approach of the Rassias theorem [34] in which he replaced the factor $\|x\|^{p}+\|y\|^{p}$ by $\|x\|^{p}\|y\|^{q}$ for $p, q \in R$ 
M.Arunkumar, P.Agilan and S.Ramamoorthy

with $p+q=1$. A generalization of the Rassias theorem was obtained by Gavruta [21] by replacing the unbounded Cauchy difference by a general control function in the spirit of Rassias approach. In 2008, a special case of Gavruta's theorem for the unbounded Cauchy difference was obtained by Ravi et.al., [38] by considering the summation of both the sum and the product of two $p-$ norms in the sprit of Rassias approach. The stability problems of several functional equations have been extensively investigated by a number of authors and there are many interesting results concerning this problem (see $[3,7,8,9$, $10,30,20,38])$.

The solution and stability of the following additive functional equations

$$
f(x+y)=f(x)+f(y)
$$

$$
f\left(n x_{0}+\sum_{i=1}^{n} x_{i}\right)=\sum_{i=1}^{n} f\left(x_{0}+x_{i}\right),
$$

$$
\begin{aligned}
& \sum_{i=1}^{n} g\left(\sum_{j=1}^{i} x_{j}\right)=\sum_{i=1}^{n}(n-i+1) g\left(x_{i}\right), n \geq 2 \\
& \sum_{i=1}^{n} p_{i} f\left(x_{i}\right)=f\left(\sum_{i=1}^{n} p_{i} x_{i}\right)
\end{aligned}
$$

were discussed in (see $[4,5,39])$

In this paper, the authors investigate the general solution and generalized UlamHyers stability of a new type of $n$ - dimensional additive functional equation of the form

$$
\begin{array}{r}
f\left(\sum_{k=1}^{n} k x_{k}\right)+\sum_{l=2}^{n} f\left(\sum_{k=1, k \neq l}^{n} k x_{k}-l x_{l}\right)+f\left(x_{1}-\sum_{k=2}^{n} k x_{k}\right) \\
=(n+1) f\left(x_{1}\right)+(n-3) \sum_{k=2}^{n} k f\left(x_{k}\right)
\end{array}
$$

With $n>3$ in Banach space and Banach Algebra using direct and fixed point methods.

Now we will recall the fundamental results in fixed point theory.

Theorem 1.1. [16] (The alternative of fixed point) Suppose that for a complete generalized metric space $(X, d)$ and a strictly contractive mapping $T: X \rightarrow X$ with Lipschitz constant $L$. Then, for each given element $x \in X$, either

$$
\text { (A1) } d\left(T^{n} x, \mathrm{~T}^{n+1} x\right)=\infty \quad \forall n \geq 0,
$$

or

(A2) there exists a natural number $n_{0}$ such that:

(i) $d\left(T^{n} x, T^{n+1} x\right)<\infty$ for all $n \geq n_{0}$;

(ii)The sequence $\left(T^{n} x\right)$ is convergent to a fixed point $y^{*}$ of $T$

(iii) $y^{*}$ is the unique fixed point of $T$ in the set $Y=\left\{y \in X: d\left(T^{n 0} x, y\right)<\infty\right\}$;

(iv) $d\left(y^{*}, y\right) \leq \frac{1}{1-L} d(y, T y)$ for all $y \in Y$. 
Solution and Generalized Ulam-Hyers Stability of a $n$-Dimensional Additive Functional Equation in Banach Space and Banach Algebra: Direct and Fixed Point Methods

\section{General solution}

In this section, the authors discuss the general solution of the functional equation (1.5) by considering $X$ and $Y$ are real vector spaces.

Theorem 2.1. If $f: X \rightarrow y$ satisfies the functional equation (1.1) for all $x, y \in X$ if and only if $f$ satisfies the functional equation (1.5) for all $x_{1}, x_{2}, x_{3}, \ldots, x_{n} \in X$.

Proof: Let $f: X \rightarrow Y$ satisfy the functional equation (1.1). Setting $x=y=0$ in (1.1), we have $f(0)=0$. Set $x=-y$ in (1.1), we get $f(-y)=-f(y)$ for all $y \in X$. Therefore $f$ is an odd function. Replacing $y$ by $x$ and $y$ by $2 x$ in (1.1), we obtain

$$
f(2 x)=2 f(x) \quad \text { and } \quad f(3 x)=3 f(x)
$$

for all $x \in X$. In general for any positive integer a, we have

$$
f(a x)=a f(x)
$$

for all $x \in X$. Replacing $x$ by $\frac{x}{a}$ in (2.2), we get

$$
f\left(\frac{x}{a}\right)=\frac{1}{a} f(x)
$$

for all $x \in X$. It is easy to verify from (1.1) that

$$
f\left(x_{1}+x_{2}+\ldots+x_{n}\right)=f\left(x_{1}\right)+f\left(x_{2}\right)+\ldots+f\left(x_{n}\right)
$$

for all $x_{1}, x_{2}, x_{3}, \ldots, x_{n} \in X$. Replacing $\left(x_{1}, x_{2}, \ldots, x_{n}\right)$ in $\left(x_{1}, 2 x_{2}, \ldots, \mathrm{n} x_{n}\right)$ in (2.4) we arrive $f\left(x_{1}, 2 x_{2}, 3 x_{3}, \ldots, \mathrm{n} x_{n}\right)=f\left(x_{1}\right)+f\left(2 x_{2}\right)+f\left(3 x_{3}\right)+\ldots+f\left(n x_{n}\right)$

for all $x_{1}, x_{2}, \ldots, x_{n} \in X$. Replacing $x_{2}$ by $-2 x_{2}, x_{3}$ by $-3 x_{3}, \ldots$, and $x_{n}$ by $-n x_{n}$ respectively in (2.4) and using the oddness of $f$, we get the following equations

$\left\{\begin{array}{l}f\left(x_{1}-2 x_{2}+3 x_{3}+\ldots+n x_{n}\right)=f\left(x_{1}\right)-f\left(2 x_{2}\right)+f\left(3 x_{3}\right)+\ldots+f\left(n x_{n}\right) \\ f\left(x_{1}+2 x_{2}-3 x_{3}+\ldots+n x_{n}\right)=f\left(x_{1}\right)+f\left(2 x_{2}\right)-f\left(3 x_{3}\right)+\ldots+f\left(n x_{n}\right) \\ f\left(x_{1}+2 x_{2}+3 x_{3}+\ldots-n x_{n}\right)=f\left(x_{1}\right)+f\left(2 x_{2}\right)+f\left(3 x_{3}\right)+\ldots-f\left(n x_{n}\right)\end{array}\right.$

for all $x_{1}, x_{2}, \ldots, x_{n} \in X$. Replacing $\left(x_{1}, x_{2}, \ldots, x_{n}\right)$ in $\left(x_{1},-2 x_{2}, \ldots,-\mathrm{n} x_{n}\right)$ in (2.4), we have

$f\left(x_{1}-2 x_{2}+3 x_{3}+\ldots+n x_{n}\right)=f\left(x_{1}\right)-f\left(2 x_{2}\right)-f\left(3 x_{3}\right)+\ldots-f\left(n x_{n}\right)$

for all $x_{1}, x_{2}, \ldots, x_{n} \in X$. Adding (2.5),(2.6), (2.7) and using (2.1) (2.2), we have

demonstrated our result.

Conversely, Let $f: X \rightarrow Y$ satisfy the functional equation (1.5). Setting $x_{1}, x_{2}, \ldots, x_{n} \in X$. by $(0,0, \ldots, 0)$ in $(1.5)$, we get $f(0)=0$. Replacing $\left(x_{1}, x_{2}, \ldots, x_{n}\right)$ by $(0, x, \ldots, 0)$ and $(x, x, \ldots, 0)$ in $(1.5)$, we obtain

$f(2 x)=2 f(x) \quad$ and $\quad f(3 x)=3 f(x)$

for all $x \in X$. Replacing $\left(x_{1}, x_{2}, \ldots, x_{n}\right)$ by $(0,-\mathrm{x}, \ldots, 0)$ and using $(2.8)$ in (1.5), we get 
M.Arunkumar, P.Agilan and S.Ramamoorthy

$f(-x)=-f(x)$ for all $x \in X$. Therefore $\mathrm{f}$ is an odd function. Replacing $\left(x_{1}, x_{2}, \ldots, x_{n}\right)$ by $\left(x_{1}, \frac{x_{2}}{2}, \ldots, 0\right)$ and using (2.8) in (1.5), we have

$$
(n-1) f\left(x_{1}+x_{2}\right)+2 f\left(x_{1}-x_{2}\right)=(n+1) f\left(x_{1}\right)+(n-3) f\left(x_{2}\right)
$$

for all $x_{1}, x_{2}, \ldots, x_{n} \in X$. Replacing $x_{1}$ by $x_{2}, x_{2}$ by $x_{1}$ in (2.9) and using the oddness of $f$, we get

$$
(n-1) f\left(x_{1}+x_{2}\right)-2 f\left(x_{1}-x_{2}\right)=(n-3) f\left(x_{1}\right)+(n+1) f\left(x_{2}\right)
$$

for all $x_{1}, x_{2}, \in X$. Adding (2.9) (2.10) and replacing $x_{1}$ by $x, x_{2}$ by $y$, and since $n>3$, we arrive our result. Hence the proof is completed.

\section{Generalized ulam-hyers stability in Banach space}

In this section, let $Y_{1}$ be a normed space and $Y_{2}$ be a Banach space. The authors investigate the generalized Ulam-Hyers stability of the $n$ - Dimensional Additive Functional equation (1.5). Define a mapping $D f: X^{n} \rightarrow Y$ by

$$
\begin{aligned}
D f\left(x_{1}, x_{2}, \ldots, x_{n}\right)=f\left(\sum_{k=1}^{n} k x_{k}\right) & +\sum_{l=2}^{n} f\left(\sum_{k=1, k \neq 1}^{n} k x_{k}-l x_{l}\right)+f\left(x_{1}-\sum_{k=2}^{n} k x_{k}\right) \\
& -(n+1) f\left(x_{1}\right)-(n-3) \sum_{k=2}^{n} k f\left(x_{k}\right)
\end{aligned}
$$

for all $x_{1}, x_{2}, \ldots, x_{n} \in X$ with $n>3$

\subsection{Direct method}

Theorem 3.1. Let $j \pm 1$. Let $\psi: X^{n} \rightarrow[0, \infty)$ be a function such that

$$
\lim _{l \rightarrow \infty} \frac{\psi\left(n^{l j} x_{1}, n^{l j} x_{2}, \ldots, n^{l j} x_{n}\right)}{n^{l j}}=0
$$

for all $x_{1}, x_{2}, \ldots, x_{n} \in X$ with $n>3$ let $f: X \rightarrow Y$ be a function satisfying the inequality

$$
\left\|D f\left(x_{1}, x_{2}, \ldots, x_{n}\right)\right\| \leq \psi\left(x_{1}, x_{2}, \ldots, x_{n}\right)
$$

for all $x_{1}, x_{2}, \ldots, x_{n} \in X$. Then there exists a unique function $\mathrm{A}: X \rightarrow Y$ such that

$$
\|f(x)-A(x)\| \leq \frac{1}{n(n-3)} \sum_{l=\frac{1-j}{2}}^{\infty} \frac{\Psi\left(n^{l j} x\right)}{n^{l j}}
$$

Where $\Psi\left(n^{l j} x\right)=(\underbrace{\psi(0, \ldots, 0)}_{n-1 \text { times }}, n^{l j} x)$ for all $x \in X$. The mapping $A(x)$ is defined by

$$
A(x)=\lim _{t \rightarrow \infty} \frac{f\left(n^{l j} x\right)}{n^{l j}}
$$

for all $x \in X$.

Proof. Assume $j=1$. Replacing $\left(x_{1}, x_{2}, \ldots, x_{n}\right)$ by $(\underbrace{0, \ldots, 0}_{n-1 \text { times }}, x)$ in (3.2) and using oddness of $f$, we get

$$
\|(n-3) f(n x)-n(n-3) f(x)\| \leq \psi(\underbrace{0, \ldots, 0, x)}_{n-1 \text { times }}
$$


Solution and Generalized Ulam-Hyers Stability of a $n$-Dimensional Additive Functional Equation in Banach Space and Banach Algebra: Direct and Fixed Point Methods for all $x \in X$. Dividing the above inequality by $n(n-3)$ we obtain

$$
\left\|\frac{f(n x)}{n}-f(x)\right\| \leq \frac{1}{n(n-3)} \psi(\underbrace{0, \ldots, 0}_{n-1 \text { times }}, x)
$$

for all $x \in X$. Letting $\Psi(x)=\psi(\underbrace{0, \ldots, 0}_{n-1 \text { times }}, x)$ in (3.6), we arrive

$$
\left\|\frac{f(n x)}{n}-f(x)\right\| \leq \frac{\Psi(x)}{n(n-3)}
$$

for all $x \in X$. Now replacing $x$ by $n x$ and dividing by $n$ in (3.7), we obtain

$$
\left\|\frac{f\left(n^{2} x\right)}{n^{2}}-\frac{f(n x)}{n}\right\| \leq \frac{\Psi(n x)}{n^{2}(n-3)}
$$

for all $x \in X$. Combining (3.7) and (3.8), we obtain

$$
\left\|\frac{f\left(n^{2} x\right)}{n^{2}}-f(x)\right\| \leq \frac{1}{n(n-3)}\left[\Psi(x)+\frac{\Psi(n x)}{n}\right]
$$

for all $x \in X$. In general for any positive integer $l$, we obtain that

$$
\begin{aligned}
\left\|\frac{f\left(n^{l} x\right)}{n^{l}}-f(x)\right\| & \leq \frac{1}{n(n-3)} \sum_{k=0}^{l-1} \frac{\Psi\left(n^{k} x\right)}{n^{k}} \\
& \leq \frac{1}{n(n-3)} \sum_{k=0}^{\infty} \frac{\Psi\left(n^{k} x\right)}{n^{k}}
\end{aligned}
$$

for all $x \in X$. In order to prove the convergence of the sequence $\left\{\frac{f\left(n^{l} x\right)}{n^{l}}\right\}$, replace $x$ by $n^{m} x$ and divided by $n^{m}$ in (3.10), for any $m, l>0$, we arrive

$$
\begin{aligned}
\left\|\frac{f\left(n^{l+m}\right) x}{n^{l+m}}-\frac{f\left(n^{m} x\right)}{n^{m}}\right\|=\frac{1}{n^{m}} \| & \frac{f\left(n^{l+m}\right) x}{n^{l+m}}-f\left(n^{m} x\right) \| \\
& \leq \frac{1}{n(n-3)} \sum_{k=0}^{\infty} \frac{\Psi\left(n^{l+m} x\right)}{n^{l+m}} \\
& \rightarrow 0 \text { as } m \rightarrow \infty
\end{aligned}
$$

For all $x \in X$. Hence the sequence $\left\{\frac{f\left(n^{l} x\right)}{n^{l}}\right\}$ is a chauchy sequence. Since $Y$ is complete, there exists a mapping $A: X \rightarrow Y$ such that

$$
A(x)=\lim _{l \rightarrow \infty} \frac{f\left(n^{l} x\right)}{n^{l}} \forall x \in X .
$$

Letting $l \rightarrow \infty$ in (3.10), we see that (3.3) holds for all $x \in X$. Now we need to prove $A$ satisfies (1.5), replacing $\left(x_{1}, x_{2}, \ldots, x_{n}\right)$ by $\left(n^{l} x_{1}, n^{l} x_{2}, \ldots, n^{l} x_{n}\right)$ and divide by $n^{l}$ in (3.2), we arrive 
M.Arunkumar, P.Agilan and S.Ramamoorthy

$$
\frac{1}{n^{l}}\left\|D f\left(n^{l} x_{1}, n^{l} x_{2}, \ldots, n^{l} x_{n}\right)\right\| \leq \frac{1}{n^{l}} \psi\left(n^{l} x_{1}, n^{l} x_{2}, \ldots, n^{l} x_{n}\right)
$$

for all $x_{1}, x_{2}, \ldots, x_{n} \in X$. Letting $l \rightarrow \infty$ in the above inequality, we see that

$$
\begin{aligned}
A\left(\sum_{k=1}^{n} k x_{k}\right)+\sum_{l=2}^{n} A & \left(\sum_{k=1, k \neq 1}^{n} k x_{k}-l x_{l}\right)+A\left(x_{1}-\sum_{k=2}^{n} k x_{k}\right) \\
& =(n+1) A\left(x_{1}\right)+(n-3) \sum_{k=2}^{n} k A\left(x_{k}\right)
\end{aligned}
$$

Hence $A$ satisfies (1.5) for all $x_{1}, x_{2}, \ldots, x_{n} \in X$ with $n>3$. To prove $A$ is unique, let $R(x)$ be another additive mapping satisfying (1.5) and (3.3). Then

$$
\begin{aligned}
\|A(x)-R(x)\| & \leq \frac{1}{n^{l}}\left\{\left\|A\left(n^{l} x\right)-f\left(n^{l} x\right)\right\|+\left\|f\left(n^{l} x\right)-R\left(n^{l} x\right)\right\|\right\} \\
& \leq \frac{2}{n(n-3)} \sum_{k=0}^{\infty} \frac{\Psi\left(n^{k+l} x\right)}{n^{k+l}} \\
& \rightarrow 0 \text { as } l \rightarrow \infty
\end{aligned}
$$

for all $x \in X$. Hence $A$ is unique.

For $j=-1$, we can prove the similar stability result. This completes the proof of the theorem.

The following corollary is a immediate consequence of Theorem 3.1 concerning the stability of (1.5).

Corollary 3.2. Let $\lambda$ and $s$ be nonnegative real numbers. If a function $f: X \rightarrow Y$ satisfies the inequality

$$
\left\|D f\left(x_{1}, x_{2}, \ldots, x_{n}\right)\right\| \leq\left\{\begin{array}{lr}
\lambda, & \mathrm{s} \neq 1 ; \\
\lambda \sum_{i=1}^{n}\left\|x_{i}\right\|^{s}, & \mathrm{~s} \neq \frac{1}{n} ; \\
\lambda\left\{\prod_{i=1}^{n}\left\|x_{i}\right\|^{s}+\prod_{i=1}^{n}\left\|x_{i}\right\|^{n s}\right\}, & \text {. }
\end{array}\right.
$$

for all $x_{1}, x_{2}, \ldots, x_{n} \in X$. Then there exists a unique additive function $A: X \rightarrow Y$ such that

for all $x \in X$.

$$
\|f(x)-A(x)\| \leq\left\{\begin{array}{l}
\frac{\lambda}{(n-3)|n-1|}, \\
\frac{\lambda\|x\|^{s}}{(n-3)\left|n-n^{s}\right|}, \\
\frac{\lambda\|x\|^{n s}}{(n-3)\left|n-n^{n s}\right|},
\end{array}\right.
$$

\subsection{Fixed Point Method}


Solution and Generalized Ulam-Hyers Stability of a $n$-Dimensional Additive Functional Equation in Banach Space and Banach Algebra: Direct and Fixed Point Methods

In this section, the authors present the generalized Ulam-Hyers stability of the functional equation (1.5) in Banach space using fixed point method.

Here after through out this section, let $V$ be a vector space and B Banach space respectively. Define a mapping

$$
\begin{aligned}
D f\left(x_{1}, x_{2}, \ldots, x_{n}\right)= & f\left(\sum_{k=1}^{n} k x_{k}\right)+\sum_{l=2}^{n} f\left(\sum_{k=1, k \neq 1}^{n} k x_{k}-l x_{l}\right) \\
& +f\left(x_{1}-\sum_{k=2}^{n} k x_{k}\right)-(n+1) f\left(x_{1}\right)-(n-3) \sum_{k=2}^{n} k f\left(x_{k}\right)
\end{aligned}
$$

for all $x_{1}, x_{2}, \ldots, x_{n} \in X$. with $n>3$.

For to prove the stability result we define the following: $\mu_{i}$ is a constant such that

$$
\mu_{i}^{k}=\left\{\begin{array}{lll}
n, & \text { if } & i=0, \\
\frac{1}{n}, & \text { if } & i=1
\end{array}\right.
$$

and $\Omega$ is the set such that

$$
\Omega=\{g \mid g: X \rightarrow Y, g(0)=0\}
$$

Theorem 3.3. Let $f: V \rightarrow B$ be a mapping for which there exist a function $\psi, \Psi, \Upsilon: V^{n} \rightarrow[0, \infty)$ with the condition

$$
\lim _{k \rightarrow \infty} \frac{1}{\mu_{i}^{k}} \psi\left(\mu_{i}^{k} x_{1}, \mu_{i}^{k} x_{2}, \ldots, \mu_{i}^{k} x_{n},\right)=0
$$

Such that the functiona inequality with

$$
\left\|D f\left(x_{1}, x_{2}, \ldots, x_{n}\right)\right\| \leq \psi\left(x_{1}, x_{2}, \ldots, x_{n}\right)
$$

for all $x_{1}, x_{2}, \ldots, x_{n} \in V$.If there exists $L=L(i)<1$. Such that the function

$$
x \rightarrow \Upsilon(x)=\frac{1}{(n-3)} \Psi\left(\frac{x}{n}\right)
$$

has the property

$$
\Upsilon(x)=L \mu_{i} \Upsilon\left(\frac{x}{\mu_{i}}\right)
$$

for all $x \in V$. Then there exists a unique additive mapping $A: V \rightarrow B$ satisfying the functional equation (1.5) and

$$
\|f(x)-A(x)\| \leq \frac{L^{1-i}}{1-L} \Upsilon(x)
$$

for all $x \in V$.

Proof. Consider the set $\Omega=\{p / p: \mathrm{X} \rightarrow Y, p(0)=0\}$ and introduce the generalized metric on $\Omega$,

$$
d(p, q)=d(p, q)=\inf \{K \in(0, \infty):\|p(x)-q(x)\| \leq K \Upsilon(x), x \in V\}
$$

It is easy to see that $(\Omega, d)$ is complete. 
M.Arunkumar, P.Agilan and S.Ramamoorthy

Define $T: \Omega \rightarrow \Omega$ by

$$
T p(x)=\frac{1}{\mu_{i}} p\left(\mu_{i} x\right),
$$

For all $x \in V$. Now, $p, q \in \Omega$, we have

$$
\begin{aligned}
d(p, q) \leq K & \\
& \Rightarrow\|p(x)-q(x)\| \leq K \Upsilon(x), x \in V \\
& \Rightarrow\left\|\frac{1}{\mu_{i}} p\left(\mu_{i} x\right)-\frac{1}{\mu_{i}} q\left(\mu_{i} x\right)\right\| \leq \frac{1}{\mu_{i}} K \Upsilon\left(\mu_{i} x\right), x \in V, \\
& \Rightarrow\left\|\frac{1}{\mu_{i}} p\left(\mu_{i} x\right)-\frac{1}{\mu_{i}} q\left(\mu_{i} x\right)\right\| \leq L K \Upsilon(x), x \in V, \\
& \Rightarrow\|T p(x)-T q(x)\| \leq L K \Upsilon(x), x \in V, \\
& \Rightarrow d(p, q) \leq L K .
\end{aligned}
$$

This implies $d(T p, T q) \leq L d(p, q)$, for all $p, q \in \Omega$. i.e., $T$ is a strictly contractive mapping on $\Omega$ with Lipschitz constant $L$. From (3.6), we arrive

$$
\left\|\frac{f(n x)}{n}-f(x)\right\| \leq \frac{1}{n(n-3)} \psi \underbrace{(0, \ldots, 0, x)}_{n-1 \text { times }}
$$

for all $x \in V$. Using (3.4) for the case $i=0$ it reduces to

$$
\|f(n x)-f(x)\| \leq \frac{1}{n} \Upsilon(x)
$$

for all $x \in V$.

$$
\text { i.e., } \quad d(T f, f) \leq \frac{1}{n}=L=L^{1-0}=L^{1-i}<\infty
$$

Again replacing $x=\frac{x}{n}$ in (3.6), we get

$$
\left\|f(x)-n f\left(\frac{x}{n}\right)\right\| \leq \frac{1}{n(n-3)} \psi(\underbrace{0, \ldots, 0, \frac{x}{n}}_{n-1 \text { titues }})
$$

for all $x \in V$. Using (3.4) for the case $i=1$ it reduces to

$$
\left\|f(x)-n f\left(\frac{x}{n}\right)\right\| \leq \Upsilon(x)
$$

for all $x \in V$.

$$
\text { i.e., } \quad d(f, T f) \leq 1=L^{0}=L^{1-1}=L^{1-i}<\infty
$$

in both cases, we arrive

$$
d(f, T f) \leq L^{1-i}
$$

Therefore (A1) holds.

By (A2), it follows that there exists a fixed point $A$ of $T$ in $\Omega$ such that

$$
A(x)=\lim _{k \rightarrow \infty} \frac{1}{\mu_{i}^{k}} f_{a}\left(\mu_{i}^{k} x\right)
$$

For all $x \in V$. 
Solution and Generalized Ulam-Hyers Stability of a $n$-Dimensional Additive Functional Equation in Banach Space and Banach Algebra: Direct and Fixed Point Methods

To prove $A: V \rightarrow B$ is additive. Replacing $\left(x_{1}, x_{2}, \ldots, x_{n}\right)$ by $\left(\mu_{i}^{k} x_{1}, \mu_{i}^{k} x_{2}, \ldots, \mu_{i}^{k} x_{n}\right)$ in (3.2) and dividing by $\mu_{i}^{k}$, it follows from (3.1) that

$$
\begin{aligned}
\left\|A\left(x_{1}, x_{2}, \ldots, x_{n}\right)\right\| & =\lim _{k \rightarrow \infty} \frac{\left\|D f\left(\mu_{i}^{k} x_{1}, \mu_{i}^{k} x_{2}, \ldots, \mu_{i}^{k} x_{n}\right)\right\|}{\mu_{i}^{k}} \\
& \leq \lim _{k \rightarrow \infty} \frac{\left\|\psi\left(\mu_{i}^{k} x_{1}, \mu_{i}^{k} x_{2}, \ldots, \mu_{i}^{k} x_{n}\right)\right\|}{\mu_{i}^{k}}=0
\end{aligned}
$$

For all $x_{1}, x_{2}, \ldots, x_{n} \in V$.i.e., A satisfies the functional equation (1.5)

By (A3), A is the unique fixed point of $T$ in the set $y=\{A \in \Omega: d(f, A)<\infty\}, A$ is the unique function such that

$$
\|f(x)-A(x)\| \leq K \Upsilon(x)
$$

for all $x \in V$ and $K>0$. Finally by (A4), we obtain

$$
d(f, A) \leq \frac{1}{1-L} d(f, T, F)
$$

this implies

Which yields

$$
d(f, A) \leq \frac{L^{1-i}}{1-L}
$$

$$
\|f(x)-A(x)\| \leq \frac{L^{1-i}}{1-L} \Upsilon(x)
$$

for all $x \in V$. This completes the proof of the theorem.

The following Corollary is an immediate consequence of Theorem (3.3) concerning the stability of (1.5)

Corollary 3.4. Let $f: V \rightarrow B$ be a mapping and there exists real numbers $\lambda$ and $s$ such that

$$
\left\|D f\left(x_{1}, x_{2}, \ldots, x_{n}\right)\right\| \leq \begin{cases}\lambda, & \mathrm{s} \neq 1 ; \\ \lambda\left\{\sum_{i=1}^{n}\left\|x_{i}\right\|^{s}\right\}, & \\ \lambda\left\{\prod_{i=1}^{n}\left\|x_{i}\right\|^{s}+\sum_{i=1}^{n}\left\|x_{i}\right\|^{n s}\right\}, & \mathrm{s} \neq \frac{1}{n} ;\end{cases}
$$


M.Arunkumar, P.Agilan and S.Ramamoorthy

for all $x_{1}, x_{2}, \ldots, x_{n} \in V$. Then there exists a unique additive function $A: V \rightarrow B$ such that

for all $x \in V$.

$$
\|f(x)-A(x)\| \leq\left\{\begin{array}{l}
\frac{\lambda}{(n-3)|n-1|}, \\
\frac{\lambda\|x\|^{s}}{(n-3)\left|n-n^{s}\right|}, \\
\frac{\lambda\|x\|^{n s}}{(n-3)\left|n-n^{n s}\right|},
\end{array}\right.
$$

\section{Proof: Setting}

$$
\psi\left(x_{1}, x_{2}, \ldots, x_{n}\right)=\left\{\begin{array}{l}
\lambda, \\
\lambda\left\{\sum_{i=1}^{n}\left\|x_{i}\right\|^{s}\right\}, \\
\lambda\left\{\prod_{i=1}^{n}\left\|x_{i}\right\|^{s}+\sum_{i=1}^{n}\left\|x_{i}\right\|^{n s}\right\},
\end{array}\right.
$$

for all $x_{1}, x_{2}, \ldots, x_{n} \in V$. Now,

$$
\begin{aligned}
& \int \frac{\lambda}{\mu_{i}^{k}}, \\
& \psi\left(\mu_{i}^{k} x_{1} \mu_{i}^{k} x_{2}, \ldots, \mu_{i}^{k} x_{n}\right)=\left\{\frac{\lambda}{\mu_{i}^{k}}\left\{\sum_{i=1}^{n}\left\|\mu_{i}^{k} x_{i}\right\|^{s}\right\},\right. \\
& \frac{\lambda}{\mu_{i}^{k}}\left\{\prod_{i=1}^{n}\left\|\mu_{i}^{k} x_{i}\right\|^{s}+\sum_{i=1}^{n}\left\|\mu_{i}^{k} x_{i}\right\|^{n s}\right\} \text {, } \\
& =\left\{\begin{array}{l}
\lambda \mu_{i}^{-k}, \\
\lambda i^{k(s-1)}\left\{\sum_{i=1}^{n}\left\|x_{i}\right\|^{s}\right\}, \\
\lambda \mu_{i}^{k(n s-1)}\left\{\prod_{i=1}^{n}\left\|x_{i}\right\|^{s}+\sum_{i=1}^{n}\left\|x_{i}\right\|^{n s}\right\},
\end{array}\right. \\
& =\left\{\begin{array}{l}
\rightarrow 0 \text { as } n \rightarrow \infty, \\
\rightarrow 0 \text { as } n \rightarrow \infty, \\
\rightarrow 0 \text { as } n \rightarrow \infty .
\end{array}\right.
\end{aligned}
$$

Thus, (3.1) is holds.

But, we have $\Upsilon(x)=\frac{1}{(n-3)} \Psi\left(\frac{x}{n}\right)=\frac{1}{(n-3)} \phi(\underbrace{0, \ldots, 0, \frac{x}{n}}_{n-1 \text { titimes }})$

Hence 
Solution and Generalized Ulam-Hyers Stability of a $n$-Dimensional Additive Functional Equation in Banach Space and Banach Algebra: Direct and Fixed Point Methods

$$
\Upsilon(x)=\frac{1}{(n-3)} \phi(\underbrace{0, \ldots, 0, \frac{x}{n}}_{n-1 \text { times }})=\left\{\begin{array}{l}
\frac{\lambda}{(n-3)}, \\
\frac{\lambda}{(n-3) n^{n}}\|x\|^{s}, \\
\frac{\lambda}{(n-3) n^{n s}}\|x\|^{n s} .
\end{array}\right.
$$

Now,

$$
\frac{1}{\mu_{i}} \Upsilon\left(\mu_{i} x\right)=\left\{\begin{array}{l}
\frac{\lambda}{\mu_{i}(n-3)}, \\
\frac{\lambda}{\mu_{i}} \frac{1}{(n-3) n^{s}}\left\|\mu_{i} x\right\|^{s}, \\
\frac{\lambda}{\mu_{i}} \frac{1}{(n-3) n^{n s}}\left\|\mu_{i} x\right\|^{n s}
\end{array} \quad=\left\{\begin{array}{l}
\mu_{i}^{-1} \gamma(x), \\
\mu_{i}^{s-1} \gamma(x), \\
\mu_{i}^{n s-1} \gamma(x) .
\end{array}\right.\right.
$$

Now from (3.5), we prove the following cases for condition (i).

Case : 1

$$
\begin{aligned}
& L=n^{-1} \text { if } i=0 \\
& \qquad\|f(x)-A(x)\| \leq \frac{\lambda}{n-3} \frac{\left(n^{-1}\right)^{1-0}}{1-n^{(-1)}}=\frac{\lambda}{(n-1)(n-3)}
\end{aligned}
$$

Case : $2 \quad L=n^{1}$ if $i=1$

$$
\|f(x)-A(x)\| \leq \frac{\lambda}{n-3}\left(\frac{\left(n^{1}\right)^{1-1}}{1-n^{1}}\right)=\frac{-\lambda}{(1-n)(n-3)}
$$

Case : $3 \quad L=n^{s-1}$ for $s<1$ if $i=0$

$$
\|f(x)-A(x)\| \leq \frac{\lambda}{(n-3) n^{s}} \frac{\left(n^{s-1}\right)^{1-0}}{1-n^{(s-1)}}\|x\|^{s}=\frac{\lambda}{(n-3)\left(n-n^{s}\right)}\|x\|^{s} .
$$

Case : $4 \quad L=\frac{1}{n^{s-1}}$ for $s>1$ if $i=1$

$$
\|f(x)-A(x)\| \leq \frac{\lambda}{n^{s}}\left(\frac{\frac{1}{n^{s-1}}}{1-\frac{1}{n^{s-1}}}\right)\|x\|^{s}=\frac{\lambda}{(n-3)\left(n^{s}-n\right)}\|x\|^{s} .
$$

Case : $5 \quad L=n^{n s-1}$ for $s<\frac{1}{n}$ if $i=0$

$$
\|f(x)-A(x)\| \leq \frac{\lambda}{(n-3) n^{n s}}\left(\frac{\left(n^{(n s-1)}\right)^{1-0}}{1-n^{(n s-1)}}\right)\|x\|^{n s}=\frac{\lambda}{(n-3)\left(n-n^{n s}\right)}\|x\|^{n s} .
$$

Case : $6 \quad L=\frac{1}{n^{n s-1}}$ for $s>\frac{1}{n}$ if $i=1$ 
M.Arunkumar, P.Agilan and S.Ramamoorthy

$$
\|f(x)-A(x)\| \leq \frac{\lambda}{n^{n s}}\left(\frac{\left(\frac{1}{n^{n s-1}}\right)^{1-1}}{1-\frac{1}{n^{n s-1}}}\right)\|x\|^{n s}=\frac{\lambda}{(n-3)\left(n^{n s}-n\right)}\|x\|^{n s} .
$$

Hence the proof is complete.

\section{Basic results in Banach algebra}

Here after, through out this paper, let us consider $B_{1}$ and $B_{2}$ to be a normed Algebra and a Banach Algebra, respectively.

Definition 4.1. $A$ C-linear mapping $A: X \rightarrow X$ is called Additive Derivation on $X$ if $A$ satisfies.

$$
A(x y)=A(x) y+x A(y)
$$

For all $x, y \in X$.

Definition 4.2. $A$ C-linear mapping $A: X \rightarrow X$ is called Generalized Additive Derivation on $X$ if $A$ satisfies.

$$
A\left(x_{1} x_{2} \ldots x_{n}\right)=A\left(x_{1}\right)\left(x_{2} \ldots x_{n}\right)+\ldots \ldots+\left(x_{1} x_{2} \ldots x_{n-1}\right) A\left(x_{n}\right)
$$

For all $x_{1} x_{2} \ldots x_{n} \in X$.

\section{Stability results: a direct method}

In this section, we investigate the generalized Ulam-Hyers stability of the functional equation (4.1)

Theorem 5.1. Let $j \pm 1$. Let $\psi: \mathrm{X}^{n} \rightarrow[0, \infty)$ be a function such that

$$
\lim _{l \rightarrow \infty} \frac{\left\|\psi\left(n^{l j} x_{1}, \mathrm{n}^{l j} x_{2}, \ldots, \mathrm{n}^{l j} x_{n}\right)\right\|}{n^{l j}}=0, \quad \lim _{l \rightarrow \infty} \frac{\left\|\psi\left(n^{l j} x_{1}, \mathrm{n}^{l j} x_{2}, \ldots, \mathrm{n}^{l j} x_{n}\right)\right\|}{n^{n l j}}=0,
$$

For all $x_{1} x_{2} \ldots x_{n} \in X$. let $\mathrm{f}: X \rightarrow Y$ be a function satisfies the inequality

$$
\left\|D f\left(x_{1}, x_{2}, \ldots, x_{n}\right)\right\| \leq \psi\left(x_{1}, x_{2}, \ldots, x_{n}\right)
$$

And

$$
\begin{array}{rl}
\| f\left(x_{1}, \ldots, x_{n-1}, x_{n}\right)-f & f\left(x_{1}\right)\left(x_{2}, \ldots, x_{n-1}, x_{n}\right)-\ldots \\
& -\left(x_{1}, x_{1}, \ldots, x_{n-1}\right) f\left(x_{n}\right) \| \leq \psi\left(x_{2}, \ldots, x_{n-1}, x_{n}\right)
\end{array}
$$

For all $x_{1} x_{2} \ldots x_{n} \in X$. Then there exists a unique function $\mathrm{A}: X \rightarrow Y$ such that

$\|f(x)-A(x)\| \leq \frac{1}{n(n-3)} \sum_{l=\frac{1-j}{2}}^{\infty} \frac{\Psi\left(n^{l j} x\right)}{n^{l j}}$

where $\Psi\left(n^{l j} x\right)=\psi(\underbrace{(0, \ldots, 0)}_{n \text {-limes }}, n^{l j} x)$ for all $x \in X$. The mapping $A(x)$ is defined by

$A(x) \lim _{l \rightarrow \infty} \frac{f\left(n^{l j} x\right)}{n^{n l j}}$ 
Solution and Generalized Ulam-Hyers Stability of a $n$-Dimensional Additive Functional Equation in Banach Space and Banach Algebra: Direct and Fixed Point Methods for all $x \in X$.

Corollary 5.2. Let $\lambda$ and $s$ be a nonnegative real numbers. If a function $f: V \rightarrow B$ satisfies the inequality

$$
\begin{aligned}
& \left\|D f\left(x_{1}, x_{2}, \ldots, x_{n}\right)\right\| \leq \begin{cases}\lambda, & \mathrm{s} \neq 1 ; \\
\sum_{i=1}^{n}\left\|x_{i}\right\|^{s}, & \\
\lambda\left\{\prod_{i=1}^{n}\left\|x_{i}\right\|^{s}+\sum_{i=1}^{n}\left\|x_{i}\right\|^{n s}\right\}, & \mathrm{s} \neq \frac{1}{n} ;\end{cases} \\
& \left\|f\left(x_{1}, \ldots, x_{n-1}, x_{n}\right)-f\left(x_{1}\right)\left(x_{2}, \ldots, x_{n-1}, x_{n}\right)-\ldots-\left(x_{1}, \ldots, x_{n-1}\right) f\left(x_{n}\right)\right\| \\
& \leq \begin{cases}\lambda, & \\
\lambda \sum_{i=1}^{n}\left\|x_{i}\right\|^{s}, & \mathrm{~s} \neq 1 ; \\
\lambda\left\{\prod_{i=1}^{n}\left\|x_{i}\right\|^{s}+\sum_{i=1}^{n}\left\|x_{i}\right\|^{n s}\right\}, & \mathrm{s} \neq \frac{1}{n} ;\end{cases}
\end{aligned}
$$

for all $x_{1}, x_{2}, \ldots, x_{n} \in V$. Then there exists a unique additive function $A: \mathrm{X} \rightarrow Y$ such that

$$
\|f(x)-A(x)\| \leq\left\{\begin{array}{l}
\frac{\lambda}{(n-3)|n-1|}, \\
\frac{\lambda\|x\|^{s}}{(n-3)\left|n-n^{s}\right|}, \\
\frac{\lambda\|x\|^{n s}}{(n-3)\left|n-n^{n s}\right|},
\end{array}\right.
$$

for all $x \in X$.

\section{Stability results: a fixed point method}

Theorem 6.1. Let $f: V \rightarrow B$ be a mapping for which there exist a function $\psi, \Psi, \gamma: V^{n} \rightarrow[0, \infty)$ with the condition

$\lim _{k \rightarrow \infty} \frac{1}{\mu_{i}^{k}} \psi\left(\mu_{i}^{k} x_{1}, \mu_{i}^{k} x_{2}, \ldots, \mu_{i}^{k} x_{n}\right)=0, \lim _{k \rightarrow \infty} \frac{1}{\mu_{i}^{k}} \psi\left(\mu_{i}^{k} x_{1}, \mu_{i}^{k} x_{2}, \ldots, \mu_{i}^{k} x_{n}\right)=0$

Such that the functional inequality with

$\left\|D f\left(x_{1}, x_{2}, \ldots, x_{n}\right)\right\| \leq \psi\left(x_{1}, x_{2}, \ldots, x_{n}\right)$

and 
M.Arunkumar, P.Agilan and S.Ramamoorthy

$$
\begin{aligned}
\| f\left(x_{1}, \ldots, x_{n-1}, x_{n}\right)- & f\left(x_{1}\right)\left(x_{2}, \ldots, x_{n-1}, x_{n}\right)-\ldots \\
& -\left(x_{1}, x_{1}, \ldots, x_{n-1}\right) f\left(x_{n}\right) \| \leq \phi\left(x_{1}, \ldots, x_{n-1}, x_{n}\right)
\end{aligned}
$$

for all $x_{1}, x_{2}, \ldots, x_{n} \in V$. If there exists $L=L(i)<1$ such that the function

$x \rightarrow \gamma(x)=\frac{1}{n-3} \Psi\left(\frac{x}{n}\right)$,

Has the property $\gamma(x)=L \mu_{i} \gamma\left(\frac{x}{\mu_{i}}\right)$,

For all $x \in X$. Then there exists a unique additive mapping $\mathrm{A}: V \rightarrow B$ satisfying the functional equation (1.5) and

$\|f(x)-A(x)\| \leq \frac{L^{1-i}}{1-L} \gamma(x) \mid$ for all $x \in X$.

Corollary 6.2. Let $\lambda$ and $s$ be a nonnegative real numbers. If a function $f: \mathrm{X} \rightarrow Y$ satisfies the inequality

$$
\begin{aligned}
& \left\|D f\left(x_{1}, x_{2}, \ldots, x_{n}\right)\right\| \leq \begin{cases}\lambda, & \multicolumn{1}{c}{\sum_{i=1}^{n}\left\|x_{i}\right\|^{s},} \\
\lambda\left\{\prod_{i=1}^{n}\left\|x_{i}\right\|^{s}+\sum_{i=1}^{n}\left\|x_{i}\right\|^{n s}\right\}, & \mathrm{s} \neq \frac{1}{n} ;\end{cases} \\
& \left\|f\left(x_{1}, \ldots, x_{n-1}, x_{n}\right)-f\left(x_{1}\right)\left(x_{2}, \ldots, x_{n-1}, x_{n}\right)-\ldots-\left(x_{1}, \ldots, x_{n-1}\right) f\left(x_{n}\right)\right\| \\
& \leq\left\{\begin{array}{l}
\lambda \sum_{i=1}^{n}\left\|x_{i}\right\|^{s}, \\
\lambda\left\{\prod_{i=1}^{n}\left\|x_{i}\right\|^{s}+\sum_{i=1}^{n}\left\|x_{i}\right\|^{n s}\right\}, \quad \mathrm{s} \neq \frac{1}{n} ;
\end{array}\right.
\end{aligned}
$$

for all $x_{1}, x_{2}, \ldots, x_{n} \in V$. Then there exists a unique additive function $A: \mathrm{X} \rightarrow Y$ such that

$$
\|f(x)-A(x)\| \leq\left\{\begin{array}{l}
\frac{\lambda}{(n-3)|n-1|}, \\
\frac{\lambda\|x\|^{s}}{(n-3)\left|n-n^{s}\right|}, \\
\frac{\lambda\|x\|^{n s}}{(n-3)\left|n-n^{n s}\right|},
\end{array} \quad \text { for all } x \in X .\right.
$$

\section{REFERENCES}

1. J. Aczel and J. Dhombres, Functional Equations in Several Variables, Cambridge Univ, Press, 1989. 
Solution and Generalized Ulam-Hyers Stability of a $n$-Dimensional Additive Functional Equation in Banach Space and Banach Algebra: Direct and Fixed Point Methods

2. T. Aoki, On the stability of the linear transformation in Banach spaces, J. Math. Soc. Japan, 2 (1950) 64-66.

3. M. Arunkumar, Solution and Stability of Arun-Additive functional equations, International Journal Mathematical Sciences and Engineering Applications, 4(3) (2010) 33-46.

4. M. Arunkumar and T.Namachivayam, stability of $n-$ homomorphisms, $n-$ derivations of a $n$-dimensional additive functional equation in $\mathrm{c}^{*}$ ternary algebras, International Journal of Advanced Computer Research, 3(3) (2013).

5. M. Arunkumar Stability of $n$ - Dimensional Additive Functional Equation in generalized $n$ - normed space, Demonstratio Mathematica, 49(3) 2016.

6. S.Czerwik, Functional Equations and Inequalities in Several Variables, World Scientiffic, River Edge, NJ,2002.

7. G.Z.Eskandani, P.Gavruta, J.M.Rassias, R.Zarghami, Generalized Hyers-Ulam Stability for a General Mixed Functional Equation in Quasi $-\beta$ - normed Spaces, Mediterr. J. Math., 8 (2011) 331-348.

8. G.Z.Eskandani, P.Gavruta, On the stability problem in quasi-Banach spaces, Nonlinear Funct, anal. Appl. (to appear).

9. P.Gavruta, A generalization of the Hyers-Ulam-Rassias stability of approximately additive mappings, J.Math. Anal. Appl., 184 (1994) 431-436.

10. P.Gavruta, An answer to a question of J.M.Rassias concerning the stability of cauchy functional equation, Advances in Equations and Inequalities, (1999) 67-71.

11. P.Gavruta, On a problem of G.Isac and Th.M.Rassias concerning the stability of mappings, J.Math. Anal. Appl., 261 (2001) 543-553.

12. D.H.Hyers, On the stability of the linear functional equation, Proc. Nat. Acad. Sci., 27 (1941) 222-224.

13. D.H.Hyers, G.Isac, Th.M.Rassias, Stability of functional equations in several variables, Birkhauser, Basel,1998.

14. S.M.Jung, Hyers-Ulam-Rassias Stability of Functional Equations in Mathematical Analysis, Hadronic Press,Palm Harbor, 2001.

15. D.O. Lee, Hyers-Ulam stability of an addtiive type functional equation, J. Appl. Math. and Computing, 13 (2003) 471-477.

16. B. Margolis, J. B. Diaz, A fixed point theorem of the alternative for contractions on a generalized complete metric space, Bull. Amer. Math. Soc., 126(74) (1968) 305-309.

17. J.M. Rassias, On approximately of approximately linear mappings by linear mappings, J. Funct. Anal. 46 (1982) 126-130.

18. J.M. Rassias, On approximately of approximately linear mappings by linear mappings, Bull. Sc. Math, 108 (1984) 445-446.

19. J.M. Rassias, H.M.Kim, Generalized Hyerslam stability for general additive functional equations in quasi $-\beta$ - normed spaces, J.Math. Anal. Appl., 356 (2009) 302-309.

20. J.M. Rassias, K.W.Jun, H.M.Kim, Approximate $(m, n)$ J.Math. Anal. Appl., 356 (2009) 302-309.

21. P. Gavruta, A generalization of the Hyers-Ulam-Rassias stability of approximately additive mappings, J.Math. Anal. Appl., 184 (1994) 431-436. 
M.Arunkumar, P.Agilan and S.Ramamoorthy

22. H. Haruki and Th.M. Rassias, New characterizations of some mean values, Journal of Mathematical Analysis and Applications, 202 (1996) 333 - 348.

23. K.W. Jun, H.M. Kim, On the Hyers-Ulam-Rassias stability of a generalized quadratic and additive type functional equation, Bull. Korean Math. Soc., 42(1) (2005) 133148.

24. K.W. Jun, H.M. Kim, On the stability of an n-dimensional quadratic and additive type functional equation, Math. Ineq. Appl., 9(1) (2006) 153-165.

25. D.H. Hyers, On the stability of the linear functional equation, Proc. Nat. Acad. Sci., 27 (1941) 222-224.

26. D.H. Hyers, G. Isac, Th.M. Rassias, Stability of functional equations in several variables, Birkhauser, Basel, 1998.

27. K.W. Jun and H.M. Kim, The generalized Hyers-Ulam-Rassias stability of a cubic functional equation, J. Math. Anal. Appl., 274 (2002) 867-878.

28. S.M. Jung, On the Hyers-Ulam stability of the functional equations that have the quadratic property, J. Math. Anal. Appl., 222 (1998) 126-137.

29. S.M. Jung, Hyers-Ulam-Rassias Stability of Functional Equations in Mathematical Analysis, Hadronic Press, Palm Harbor, 2001.

30. Pl. Kannappan, Quadratic functional equation inner product spaces, Results Math., 27(3-4) (1995) 368-372.

31. M. Moslehian and Th.M. Rassias, Stability of functional equations in nonArchimedian spaces, Applicable Analysis and Discrete Mathematics, 1(2) (2007) 110.

32. A. Najati, M.B. Moghimi, On the stability of a quadratic and additive functional equation, J. Math. Anal. Appl., 337 (2008) 399-415.

33. J.M. Rassias and M.J. Rassias, On the Ulam stability of Jensen and Jensen type mappings on the restricted domains, J. Math. Anal. Appl., 281 (2003) 516-524.

34. Th.M. Rassias, On the stability of the linear mapping in Banach spaces, Proc. Amer. Math. Soc., 72 (1978) 297-300.

35. Th.M. Rassias and P. Semrl, On the behavior of mappings which do not satisfy Hyers- Ulam stability, Proc. Amer. Math. Soc., 114 (1992) 989-993.

36. Th.M. Rassias, On the stability of functional equations and a problem of Ulam, Acta. Appl. Math., 62 (2000) 23-130.

37. Th.M. Rassias, Functional Equations, Inequalities and Applications, Kluwer Acedamic Publishers, Dordrecht, Bostan London, 2003.

38. K. Ravi, M. Arunkumar and J.M. Rassias, On the Ulam stability for the orthogonally general Euler-Lagrange type functional equation, International Journal of Mathematical Sciences, 3 (2008) 36-47.

39. J. Tipyan , C. Srisawat, P. Udomkavanich and P. Nakmahachalasint, The generalized stability of an $n$ - dimensional jensen type functional equation, Thai Journal Mathematics, 12 (2014) 265-274.

40. G. Toader and Th.M. Rassias, New properties of some mean values, Journal of Mathematical Analysis and Applications, 232 (1999) 376-383.

41. S.M. Ulam, Problems in Modern Mathematics, Science Editions, Wiley, NewYork, 1964. 